managers of where they might look for improvements, and it is precisely for this reason that accuracy is essential and that trends are monitored.

Contributors: PF coordinated the study and drafted most of the paper. AG and NR were responsible for the Oxfordshire Health Authority database. SD and RH were responsible for the Chartered Institute of Management Accountants database. PF is the guarantor.

Funding: None

Competing interests: None declared.

Fenn P, Hermans D, Dingwall R. Estimating the cost of compensatin victims of medical negligence. $B M J$ 1994;309:389-91
2 Mawhinney B. Medical negligence claims. House of Commons official report (Hansard) 1994 March 14:239. (No 1648.)

3 Towse A, Danzon P. Medical negligence and the NHS: an economic analysis. Health Econ 1999;8:93-101.

4 Toynbee P. An arm and a leg. Guardian 1999 June 25.

5 National Audit Office. Report of the comptroller and auditor general on the NHS (England) summarised accounts 1996-97. London: Stationery Office, 1998. (HC 923, 21 July 1998.)

6 Office of National Statistics. Key population and vital statistics: local and health authority areas. London: Stationery Office, 1998. (Series VS No 23, PPI No 19.)

7 Office of National Statistics. Regional trends 34. London: Stationery Office, 1999.

8 Fenn P, Diacon S, Hodges R, Watson P. Accounting for risk in the NHS Report to the Chartered Institute of Management Accountants. London: CIMA (in press).

(Accepted 22 February 2000)

\title{
Suicide by patients: questionnaire study of its effect on consultant psychiatrists
}

\author{
David A Alexander, Susan Klein, Nicola M Gray, Ian G Dewar, John M Eagles
}

\begin{abstract}
Objective To identify the effect of patients' suicide on consultant psychiatrists in Scotland.

Design Confidential coded postal questionnaire survey.

Participants Of 315 eligible consultant psychiatrists, $247(78 \%)$ contributed.

Setting Scotland.

Main outcome measures Experience of patient suicide; the features and impact of "most distressing" suicide and what helped them to deal with it.

Results 167 (68\%) consultants had had a patient commit suicide under their care. Fifty four (33\%) reported being affected personally in terms of low mood, poor sleep, or irritability. Changes in professional practice were described by 69 (42\%) of the psychiatrists-for example, a more structured approach to the management of patients at risk and increased use of mental health legislation. Twenty four $(15 \%)$ doctors considered taking early retirement because of a patient's suicide. Colleagues and family or friends were the best sources of help, and team and critical incident reviews were also useful.

Conclusions Suicide by patients has a substantial emotional and professional effect on consultant psychiatrists. Support from colleagues is helpful, and professional reviews provide opportunities for learning and improved management of suicide and its aftermath.
\end{abstract}

\section{Introduction}

Stress among doctors has been identified as an important and legitimate occupational health issue. Surveys of general practitioners, ${ }^{1}$ junior house officers, ${ }^{2}$ and hospital consultants ${ }^{3}$ have identified high levels of emotional problems, including "burnout." Consultant psychiatrists have been found to have higher levels of work related exhaustion and depression than physicians and surgeons. ${ }^{4}$ Different work stressors have been identified, including high workload and organisational conflicts, but the impact of suicide by patients, a potentially disturbing event, has not been investigated in the United Kingdom. Although the issue has been studied in the United States, Canada, and New Zealand, ${ }^{5-11}$ many of these studies are limited by the use of small or selective samples, case histories, and anecdotal evidence. We conducted a systematic inquiry into the effect of patients' suicide on a large and non-selected group of senior psychiatrists in Scotland.

\section{Participants and methods}

We sent a confidential questionnaire to all 315 consultant psychiatrists in Scotland identified through the mailing list of the Royal College of Psychiatrists and by cross checks with hospital trusts. The questionnaire was based on a review of the literature, consultation with psychiatric colleagues, and a pilot study. It comprised two sections. The first collected personal information: sex, specialty or subspecialty, number of years in psychiatry, number of years as a consultant psychiatrist, and number of suicides experienced in their consultant careers. The second section required the consultants to identify the "most distressing" suicide they had encountered as a consultant, its professional and personal impact on them, and what helped them to cope.

Throughout the questionnaire there were free text sections. To protect confidentiality, qualitative data were analysed by the non-clinical researchers (SK and NG), who were least likely to recognise any subjects from their replies.

\section{Results}

We received 247 completed questionnaires (a response rate of $78 \%$ ), but not all respondents completed all items. One hundred and fifty six $(63 \%)$ of the respondents were men. The mean number of years in psychiatry was 17.5 (SD 7.2), and the mean number of years as a consultant was 10 (SD 7.8). Sixty nine (28\%) consultants worked in general adult psychiatry, $49(20 \%)$ in old

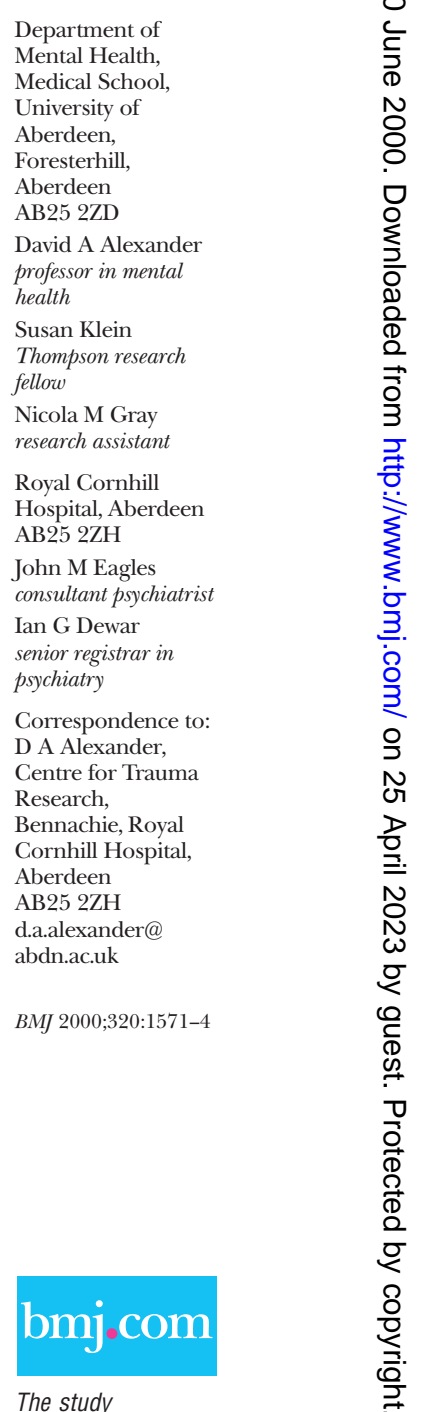
questionnaire is available on the BMJ's website 
Table 1 Perceived helpfulness of events and proceedings after suicide by patients. Values are numbers (percentages) of consultants for whom the item was applicable

\begin{tabular}{lccccccr} 
& $\begin{array}{c}\text { Very } \\
\text { helpful }\end{array}$ & Helpful & Neutral & Unhelpful & $\begin{array}{c}\text { Very } \\
\text { unhelpful }\end{array}$ & Total & $\begin{array}{c}\text { No (\%) not } \\
\text { applicable }\end{array}$ \\
\hline Fatal accident inquiry & 0 & $6(19)$ & $10(32)$ & $8(26)$ & $7(23)$ & 31 & $130(81)$ \\
\hline Disciplinary proceedings & $1(9)$ & 0 & $5(46)$ & $3(27)$ & $2(18)$ & 11 & $152(93)$ \\
\hline Legal proceedings & 0 & $2(12)$ & $1(6)$ & $3(18)$ & $11(65)$ & 17 & $146(90)$ \\
\hline Critical incident review & $14(17)$ & $42(51)$ & $18(22)$ & $8(10)$ & $1(1)$ & 83 & $81(50)$ \\
\hline Team meeting & $39(32)$ & $65(54)$ & $15(12)$ & $1(1)$ & $1(1)$ & 121 & $43(26)$ \\
\hline Funeral & $6(25)$ & $9(38)$ & $6(25)$ & $3(12)$ & 0 & 24 & $136(85)$ \\
\hline Other & $10(27)$ & $17(46)$ & $2(5)$ & $4(11)$ & $4(11)$ & 37 & $119(77)$ \\
\hline
\end{tabular}

age psychiatry; $29(12 \%)$ in rehabilitation, $26(11 \%)$ in child and adolescent psychiatry, $17(7 \%)$ in forensic psychiatry, $15(6 \%)$ in learning disabilities, $14(6 \%)$ in liaison, and $12(5 \%)$ each in psychotherapy and substance abuse. Sixteen had posts that included several specialties.

Since becoming a consultant, 167 out of 247 (67\%) reported having had a patient under their care commit suicide, but 19 consultants did not indicate how many suicides they had experienced. Of the remainder, 31 (21\%) had experienced only one suicide; $97(66 \%)$ had experienced two to six suicides, and $20(13 \%)$ had experienced seven to 15 .

Patient status and suicide characteristics

A total of 159 consultants provided information on their "most distressing" suicide. Half of the patients involved (79) were outpatients, 71 (45\%) were inpatients, seven $(4 \%)$ were day patients, and two killed themselves in prison. Twenty three of the inpatients $(32 \%)$ were compulsorily detained under mental health legislation, and only five were subject to constant or special nursing observation. Twenty six killed themselves in the ward, and four in the hospital grounds.

Eighty five patients had a history of deliberate self harm. The number of previous episodes ranged from one to 101 , with a median of two and a modal value of one.

Effects on personal lives and professional practice The interval between the suicide and the survey ranged from 1 month to 20 years (median 3 years). Fifty four $(33 \%)$ of the consultants admitted that the suicides had affected their personal lives. The most commonly reported effects were irritability at home, being less

Table 2 Perceived helpfulness to consultants of individuals and agents. Values are numbers (percentages) of consultants for whom item was applicable

\begin{tabular}{|c|c|c|c|c|c|c|c|}
\hline & $\begin{array}{l}\text { Very } \\
\text { helpful }\end{array}$ & Helpful & Neutral & Unhelpful & $\begin{array}{c}\text { Very } \\
\text { unhelpful }\end{array}$ & Total & $\begin{array}{l}\text { No }(\%) \text { not } \\
\text { applicable }\end{array}$ \\
\hline Clergy or spiritual leader & $3(21)$ & $6(43)$ & $4(29)$ & 0 & $1(7)$ & 14 & 151 (92) \\
\hline Own family or partner & $47(39)$ & $55(46)$ & $17(14)$ & $1(1)$ & 0 & 120 & $44(27)$ \\
\hline Own friend(s) & $24(34)$ & $33(47)$ & $13(19)$ & 0 & 0 & 70 & $92(57)$ \\
\hline Patient's family & $18(14)$ & $45(36)$ & $34(27)$ & $13(10)$ & $15(12)$ & 125 & $41(25)$ \\
\hline Patient's friend(s) & $4(11)$ & $9(26)$ & $13(37)$ & $5(14)$ & $4(11)$ & 35 & $129(79)$ \\
\hline Other patients & $3(6)$ & $12(25)$ & $26(53)$ & $8(16)$ & 0 & 49 & $115(70)$ \\
\hline Own general practitioner & 0 & 0 & 7 (88) & $1(12)$ & 0 & 8 & $157(95)$ \\
\hline Other team colleagues & $65(45)$ & $69(48)$ & $7(5)$ & $1(1)$ & $2(1)$ & 144 & $22(13)$ \\
\hline Other psychiatrists & $58(41)$ & $61(44)$ & $15(11)$ & $4(3)$ & $2(1)$ & 140 & $26(16)$ \\
\hline $\begin{array}{l}\text { Other mental health } \\
\text { professionals }\end{array}$ & $26(30)$ & $39(45)$ & $19(22)$ & $3(3)$ & 0 & 87 & $78(47)$ \\
\hline $\begin{array}{r}\text { Medical defence } \\
\text { organisations }\end{array}$ & $8(32)$ & $11(44)$ & $5(20)$ & $1(4)$ & 0 & 25 & $140(85)$ \\
\hline
\end{tabular}

able to deal with routine family problems, poor sleep, low mood and anhedonia, preoccupation with the suicide, and decreased self confidence. Although no consultant took time off work after the suicide, many of these effects were persistent. Among the 48 consultants who reported a time scale, four stated that the effects had lasted up to a week, 15 up to one month, 15 up to three months, and 14 over three months.

Sixty nine (42\%) consultants stated that their management of potentially suicidal patients altered after the suicide. The most commonly reported changes were more structured management, heightened awareness of suicide risk, more use of suicide observation, more detailed communication about records, more willingness to use the mental health legislation, and a more cautious and defensive approach to patients at risk. No consultant declined to take on high risk patients. The events also caused $24(15 \%)$ of the consultants to consider taking early retirement; five considered it "seriously."

\section{Factors which exacerbated or modulated the effect} Of the 56 consultants who had been aware of publicity in the media about the suicide, eight found the publicity extremely distressing and 19 found it moderately distressing. Twenty one of the 159 were moderately distressed at the prospect of litigation and 12 were extremely distressed.

Table 1 describes the extent to which the consultants found events and proceedings after the suicide to be helpful. Team meetings were commonly held and found to be helpful. Critical incident reviews occurred after only half the suicides but were viewed as helpful. Such events often led to improved management of suicide and its aftermath. Twenty four consultants attended the patient's funeral; 15 (63\%) of them found this helpful.

Legal and disciplinary proceedings and fatal accident inquiries, although uncommon, were generally viewed unfavourably. The most common event described under the "other" category was the intervention of the Mental Welfare Commission. This too was generally found to be unhelpful. Open text observations regarding the commission, inquiries, and legal and disciplinary proceedings included many statements relating to "scapegoats," "the blame culture," and "witch hunting."

Team members, other psychiatrists, and the consultants' own families and friends emerged as the most common and effective sources of help after patient suicide (table 2). General practitioners and spiritual leaders or clergy were rarely consulted. Medical defence organisations were infrequently used, but, when consulted, they were generally found to be helpful. Patients' families and friends were regarded as unhelpful by about a quarter of the consultants.

\section{Discussion}

We conducted a survey of the effect of suicide by patients in a large representative sample of consultant psychiatrists in Scotland. We studied the most distressing suicides the consultants had encountered, and their reported experiences therefore may not be representative of all suicides with which they had had to deal. The high response rate $(78 \%)$ and the extensive and frank 
open text comments confirmed the importance of this study to participants. This contrasts with the low response rates and defensiveness of psychiatrists described in earlier surveys. ${ }^{12}$

\section{Prevention and prediction}

Psychiatrists have to strike a difficult balance in their attitudes to suicide. If they regard suicide as fundamentally unavoidable it may shield the profession from blame, but such a belief may foster therapeutic nihilism. ${ }^{13}$ On the other hand, if suicide is perceived to be largely preventable and predictable, this may foster a culture of blame (as some consultants had unhelpfully experienced). ${ }^{14}$ The "blame" may be self blame, as expressed by one consultant: "There is a terrible sense of failure at having let down those who have put their trust in you."

Morris identified the unexpectedness of suicide as the most traumatising feature. ${ }^{15}$ The details of the suicides provided by the consultants in our study suggest that many were relatively unexpected; half of the suicides involved outpatients, few of the inpatients had been under special observation, and half of patients had made no previous effort at self harm.

In this survey a third of the inpatients killed themselves in their own ward, which is likely to have an adverse effect on staff and patients. This creates an additional pressure on consultants, who have a responsibility for the welfare of these groups too. It is reassuring, however, that $31 \%(15 / 49)$ consultants reported that other patients had been helpful to them after patient suicide, with only eight (16\%) consultants claiming that other patients had been unhelpful.

\section{Coping after suicide}

Some authorities have commented on the value of the "rituals of death," including attending patients' funerals after suicide. ${ }^{11}$ Only $24(15 \%)$ of the psychiatrists, however, attended their patient's funeral (although most of them who did found this helpful). The low number attending funerals may reflect a degree of denial and avoidance or an anxiety about the reactions of the patients' families.

Twenty four psychiatrists considered taking early retirement after the incident. In their questionnaire survey of consultant psychiatrists who had sought early retirement, Kendell and Pearce did not find that patient suicide was a primary reason for early retirement. ${ }^{16}$

There was a move towards more cautious practices with regard to patients at risk. This trend has been reported elsewhere after suicide. . $^{7-12}$

Support from colleagues helped to mitigate the sense of isolation some doctors reported after a patient's suicide. Outside the professional realm, doctors' families and friends proved helpful, although they must also have been under strain because of the personal changes the consultants reported in themselves and in their relationships. Few consultants consulted their general practitioners, which might suggest that their distress did not develop into a more serious health issue. Alternatively, it might reflect the widely observed reluctance of doctors to seek help.

The psychiatrists held mixed views about the helpfulness of patients' families and friends. They need to
What is already known on this topic

Consultant psychiatrists experience high levels of work related stress

Suicide by patients has been shown to cause stress but has not been systematically studied in Britain

\section{What this study adds}

Suicide by patients is genuinely distressing for consultant psychiatrists, affecting their work and relationships

Support from friends, family, and colleagues is particularly helpful, as are team and critical incident reviews

Formal inquiries were generally considered unhelpful because they often created a climate of blame

be prepared for and learn how to deal with adverse reactions from such people.

\section{Team reviews, critical incident reviews, and other} formal proceedings

Reviews were found helpful by almost all consultants. This contrasted with the negative views of legal proceedings, fatal accident inquiries, the involvement of the Mental Welfare Commission, and trust disciplinary proceedings. Others have commented on the healing potential of formal inquiries provided that they are not conducted when participants are still in an emotional turmoil. ${ }^{11}{ }^{13}{ }^{18}$ Such inquiries need to be conducted in a constructive climate and geared towards learning rather than blaming. The "blame culture" created additional distress for consultants and impeded a constructive analysis of the incident. Legal and managerial staff need to meet the challenge of creating a favourable climate for conducting inquiries into suicide and other adverse events.

Some consultants emphasised the need for the inevitability (rather than the risk) of suicide to be acknowledged in training in order that staff can be better prepared for such an event. The need for better training was also identified in a recent survey of trainee psychiatrists. $^{19}$

The consultants also highlighted the need for a balance between ensuring the availability of support and more formal methods of help (such as counselling and critical incident debriefing) and staff not being coerced into taking up such offers. This issue is pertinent in view of the lack of rigorous assessment of the prophylactic value of such interventions. ${ }^{20}$

Other important observations were that the reviews should be "clinically led" and well structured and that legal consequences should be separated from critical incident analysis. Most consultants emphasised the need to be realistic, a point cogently made by one of them: "As doctors we have high expectations of ourselves, and it is sometimes important to recalibrate these in terms of what we can realistically achieve."

The views expressed in this paper are those of the authors and do not necessarily reflect those of the funding body. We thank all the consultants who contributed to this survey. 
Contributors: DAA and JME were instrumental in formulating the idea for the survey, and this was further developed in collaboration with IGD. All authors contributed to its design, the preparation of the questionnaire, and the identification of consultant psychiatrists in Scotland. SK and NMG carried out the pilot study, took responsibility for data entry and analysis, and advised on the interpretation of the data. DAA and JME were responsible for initial drafting of the paper, but all authors contributed to the final version. DAA and JME are the guarantors.

Funding: Grampian Healthcare NHS Trust.

Competing interests: None declared.

1 Royal College of General Practitioners. Stress management in general practice. London: RCGP, 1993. (Occasional paper 61.)

Firth-Cozens J, Morrison LA. Sources of stress and ways of coping in junior house officers. Stress Medicine 1989;5:121-6.

3 Ramirez AJ, Graham J, Richards MA, Cull A, Gregory WM. Mental health of hospital consultants: the effects of stress and satisfaction at work. Lancet 1996:347:724-8.

4 Deary IJ, Agius RM, Sadler A. Personality and stress in consultant psychiatrists. Int J Soc Psychiatry 1996;42:112-23.

5 Chemtob CM, Hamada RS, Bauer G, Kinney B, Torigoe RY. Patients' suicide: frequency and impact on psychiatrists. Am J Psychiatry 1988; 145:224-8.

6 Litman RE. When patients commit suicide. Am J Psychiatry 1965;19: 570-84.

7 Menninger WW. Patient suicide and its impact on the psychotherapist. Bull Menninger Clin 1991;55:216-27.
8 Gralnick A. Suicide in the psychiatric hospital. Child Psychiatry Hum Dev 1993;24:3-12.

9 Kaye NS, Soreft SM. The psychiatrist's role, responses, and responsibilities when a patient commits suicide. Am J Psychiatry 1991;148:739-43.

10 O'Reilly RL, Truant GS, Donaldson L. Psychiatrists' experience of suicide in their patients. Psychiatry J Univ Ottawa 1990;15:173-6.

11 Little JD. Staff response to in-patient and out-patient suicide: what happened and what do we do? Aust N Z J Psychiatry 1992;26:162-7.

12 Goldstein LS, Buongiorno PA. Psychotherapists as suicide survivors. Am J Psychiatry 1984;38:392-8.

13 Bartels SJ. The aftermath of suicide on the psychiatric in-patient unit. Gen Hosp Psychiatry 1987;9:189-97.

14 Reder P, Duncan S. Reflections on child abuse inquiries. In: Peay J, ed. Inquiries after homicide. London: Duckworth, 1996.

15 Morris M. The aftermath of suicide. Br J Nurs 1995;4:205-8

16 Kendell RE, Pearce A. Consultant psychiatrists who retired prematurely in 1995-1996. Psychiatr Bull 1997;21:741-5.

17 Eastman N. Inquiry into homicides by psychiatric patients: systematic audit should replace mandatory inquiries. $B M J 1996 ; 313: 1069-71$.

18 Schneidman ES. The management of the pre-suicidal, suicidal, and postsuicide patient. Ann Intern Med 1971;75:441-58.

19 Dewar I, Eagles JM, Klein S, Gray N, Alexander DA. Psychiatric trainees experience of, and reactions to, patient suicide. Psychiatr Bull 1999;24:20-3.

20 Alexander DA. Human reactions to trauma. In: Greaves I, Porter KM, eds Pre-hospital medicine. Principles and practice of immediate care. London: Arnold, 1999

(Accepted 24 February 2000)

\title{
Empirical assessment of effect of publication bias on meta-analyses
}

\author{
A J Sutton, S J Duval, R L Tweedie, K R Abrams, D R Jones
}

\section{Department of \\ Epidemiology and \\ Public Health, \\ University of \\ Leicester, Leicester \\ LE1 6TP \\ A J Sutton \\ lecturer in medical \\ statistics \\ K R Abrams \\ senior lecturer in \\ medical statistics \\ D R Jones \\ professor in medical \\ statistics \\ Division of \\ Epidemiology, \\ School of Public \\ Health, University \\ of Minnesota, \\ Minneapolis, USA \\ S J Duval \\ assistant professor \\ continued over}

BMJ 2000;320:1574-7

\section{bmj.com}

Figures illustrating the method and funnel plots of all trials and a full table of results are available on the BMJ's website

\begin{abstract}
Objective To assess the effect of publication bias on the results and conclusions of systematic reviews and meta-analyses.

Design Analysis of published meta-analyses by trim and fill method.

Studies 48 reviews in Cochrane Database of Systematic Reviews that considered a binary endpoint and contained 10 or more individual studies. Main outcome measures Number of reviews with missing studies and effect on conclusions of meta-analyses.

Results The trim and fill fixed effects analysis method estimated that $26(54 \%)$ of reviews had missing studies and in 10 the number missing was significant. The corresponding figures with a random effects model were 23 (48\%) and eight. In four cases, statistical inferences regarding the effect of the intervention were changed after the overall estimate for publication bias was adjusted for.
\end{abstract}

Conclusions Publication or related biases were common within the sample of meta-analyses assessed. In most cases these biases did not affect the conclusions. Nevertheless, researchers should check routinely whether conclusions of systematic reviews are robust to possible non-random selection mechanisms.

\section{Introduction}

Selection bias is known to occur in meta-analyses because studies with results that are significant, interesting, from large well-funded studies, or of higher quality are more likely to be submitted, published, or published more rapidly than work without such characteristics. ${ }^{1}$ A meta-analysis based on a literature search will thus include such studies differentially, and the resulting bias may invalidate the conclusions.

The best way to deal with these problems, which we shall collectively label "publication bias," is to avoid them. Recently, for example, a trial amnesty was announced that encouraged researchers to submit for publication reports of previously unpublished trials. ${ }^{2}$ Additionally, steps are being taken to encourage the prospective registration of trials through trial registries. ${ }^{3}$ Although these steps may reduce the problem of publication bias in the future, it will remain a serious problem, and one that meta-analysts need to address for some time to come.

The simplest and most commonly used method to detect publication bias is an informal examination of a funnel plot. ${ }^{4}$ Formal tests for publication bias, such as those developed by Begg and Mazumdar ${ }^{5}$ and Egger et $\mathrm{al}^{6}{ }^{6}$ exist, but in practice few meta-analyses have assessed or adjusted for the presence of publication bias. A recent assessment of the quality of systematic reviews reported that only $6.5 \%$ and $3.2 \%$ of studies in high impact general and specialist journals respectively reported that a funnel plot had been examined. ${ }^{7}$ The uptake of any formal methods is lower still, although there are notable exceptions. ${ }^{8}$

The main aim of this paper is to assess what effect publication bias could have on the results and conclusions of meta-analyses of randomised controlled 